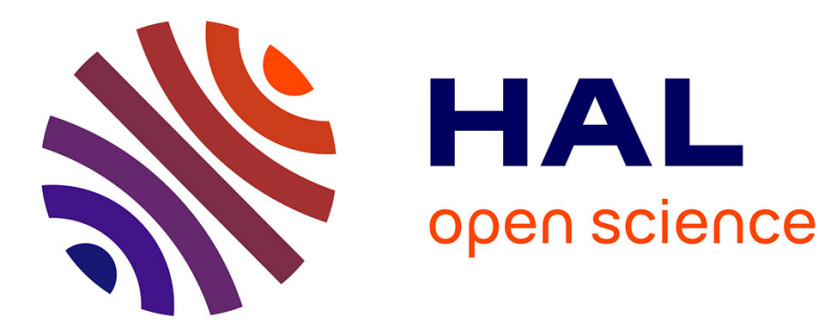

\title{
Superfocusing and Light Confinement by Surface Plasmon Excitation Through Radially Polarized Beam
}

\author{
F.I. Baida, A. Belkhir
}

\section{To cite this version:}

F.I. Baida, A. Belkhir. Superfocusing and Light Confinement by Surface Plasmon Excitation Through Radially Polarized Beam. Plasmonics, 2009, 4 (1), pp.51-59. 10.1007/s11468-008-9076-9 . hal00369215

\section{HAL Id: hal-00369215 \\ https://hal.science/hal-00369215}

Submitted on 6 May 2021

HAL is a multi-disciplinary open access archive for the deposit and dissemination of scientific research documents, whether they are published or not. The documents may come from teaching and research institutions in France or abroad, or from public or private research centers.
L'archive ouverte pluridisciplinaire HAL, est destinée au dépôt et à la diffusion de documents scientifiques de niveau recherche, publiés ou non, émanant des établissements d'enseignement et de recherche français ou étrangers, des laboratoires publics ou privés. 


\title{
Superfocusing and Light Confinement by Surface Plasmon Excitation Through Radially Polarized Beam
}

\author{
F. I. Baida · A. Belkhir
}

\begin{abstract}
We theoretically demonstrate the possibility of obtaining nanosources through an original schema based on the generation of the radially polarized surface plasmon mode of a cylindrical metallic tip. This mode has no cutoff radius and can propagate along the tip walls until its nanometric-sized apex. Instead of radiating from the tip end, the guided mode will give rise to a nanospotlight via the well-known antenna effect. 3D calculations demonstrate that both surface plasmon-guided mode and antenna effect are directly involved in the light confinement. Near-field optical microscopy can benefit significantly from this kind of probe because the sample does not need to be directly illuminated.
\end{abstract}

Keywords Surface plasmon • Guide • Taper •

Antenna effect $\cdot$ Nanofocus $\cdot$ Near field .

Nanophotonics $\cdot$ Diffraction $\cdot$ Microscope probe

\section{Introduction}

As it is well admitted now, the scanning near-field optical microscope (SNOM) leads to images that are very hard to be interpreted $[1,2]$. However, the use of

F. I. Baida $(\bowtie) \cdot$ A. Belkhir

Département d'Optique P.M. Duffieux, Institut FEMTO-ST, UMR 6174 CNRS, Université de Franche-Comté,

16 route de Gray, 25030 Besanon cedex, France

e-mail: fadi.baida@univ-fcomte.fr

\section{A. Belkhir}

Laboratoire de Physique et Chimie Quantique,

Université Mouloud Mammeri,

Tizi-Ouzou, Algeria such microscope keeps growing during the last 10 years especially due to its ability to make very local detection of the light distribution surrounding very small samples. Thus, quantum dots [3-6], photonic crystals [7-13], or nonlinear materials (Raman, fluorescence, ...) [14-17] are currently characterized thanks to optical near-field microscopes working with conventional tips (coated or uncoated tapered optical fibers). Apertureless nearfield optical microscopes are often used by exploiting the tip effect, also named the "antenna effect", that leads to a large light confinement at the tip apex (see [18] and references therein). In that case, the confinement is generated by directly illuminating the end part of the probe with a linearly polarized optical beam having an electric field with nonzero component along the tip axis. Thus, the sample is also illuminated over a very large area. Consequently, parasitical optical reflections could contaminate the recorded local optical signal.

To enhance the resolution of the optical near-field microscope, it is necessary to decrease the size of the active zone of the used probe. By active zone, we mean the effective part of the tip that is involved in the detection (for the collection mode) or the illumination (when the tip is used as a local source) processes. Several ideas were proposed [19-22] to enhance this resolution. In most of the cases, a resonance is used to locally enhance the collected or the emitted signals.

Recently, nanostructured probes were proposed and theoretically studied. In [23], corrugations (semicircular or oval shape) are made on the inner dielectric-metal interface of the fiber before the metal coating. It is demonstrated that gain of transmission depends on the position of the corrugation with respect to the tip end. Let us mention that 2D-FDTD numerical simulations are performed in that paper. 
In [24], Ding et al. demonstrated a superfocusing phenomenon that is obtained thanks to a Surface plasmon polariton (SPP) excitation. This latter is generated by modifying the geometry of the apertureless tip end. By this way, the overlap between the radially polarized guided mode of the fiber and the radially polarized SPP is enhanced. The originality of that study is the use of the $\mathrm{TM}_{01}$-guided mode of the optical fiber for the excitation of the SPP. This latter has no cutoff and can propagate whatever is the value of the metal cylinder radius. A complete study on the excitation, the propagation, and the radiation of this SPP mode is presented in that paper.

A similar study was also presented by Chen et al. in a recent paper [25], where the radially polarized SPP mode of a coated tapered optical fiber is used to obtain a highly confined electric field. Another interesting experimental study is presented in [26], where only the half of the tip is structured (over one side) with a semicircular grating. Experimental demonstration of the SPP generation and propagation toward the probe apex is clearly shown in that paper.

We present here another alternative to get high powered nanosources based on light confinement at the apex of an apertureless metallic tip. For this purpose, we combine two effects: the excitation and the propagation of the radially polarized SPP mode that has no cutoff together with the well-known antenna effect appearing at the tip apex. As in [27], where the finite element method (FEM) is used to simulate the propagation of surface plasmon polariton tip mode as it travels toward the taper of a conventional metallic tip, we perform 3D calculations using the body-ofrevolution finite time difference domain (BOR-FDTD) in the case of a structured tip illuminated by a radially polarized beam.

\section{The antenna effect}

This phenomenon has been widely theoretically studied and experimentally used during the last decade. Exhaustive bibliography can be found in the book of Shalaev and Kawata [18]. For the theoretical studies, 2D calculations have been done leading to the consideration of a prismatic tip instead of the 3D conical one [28-30]. Other works are based on 3D calculations but, according to us and in most of them, the tip description is questionable. In fact, modest tips (smaller than $1 \mu \mathrm{m}$ length) are often considered and they are supposed to be illuminated by a plane wave [31-36]. Consequently, it is clear that the "upper" nonphysical ending of the tip will contaminate the signal at its apex.
Some other studies, qualified by a nonrigorous, are based on semianalytical models such as the quasi-static approach $[37,38]$ and give a very clear insight on this phenomenon.

In all cases, it is demonstrated that the antenna effect can be induced with the axial component (along the tip axis) of the illuminating electric field. In order to optimize this effect and keep the axial symmetry for the illumination, it is clear that a radially polarized beam is perfectly adapted. In addition, after shaping (through conventional optical components) and converging towards its axis of symmetry, this conical light beam turns into Bessel beam that only presents axial electric field at its center [39]. So, the apex of the tip must be placed at this peculiar location to get efficient excitation of the antenna effect.

Our aim here is not to study the antenna effect itself but to point out the conditions to fulfill in order to get benefit from it and to clarify the origin of the expected confinement of the radially polarized surface plasmonguided mode. Thus, we do not present an extensive study of this effect but only a spectral study that demonstrates the occurring of this effect at the apex of our considered tip.

Consequently, our first study is limited to the determination of the light distribution surrounding the apex of a metallic tip illuminated by a beam having a nonzero axial component of the electric field. For this purpose, we use a home-made BOR-FDTD code. The cylindrical symmetry of the structure is fully exploited [40] leading to 2D calculations instead of 3D ones [41, 42]. Thanks to a nonuniform mesh grid, a calculation window of $60 \times 9 \mu \mathrm{m}^{2}$ is considered allowing to take into account a very long and realistic tip $\left(52 \mu \mathrm{m}\right.$ length and $10^{\circ}$ for the half-cone angle).

For this purpose, the structure is meshed with a spatial resolution varying from $\delta_{\mathrm{r}}=\delta_{\mathrm{z}}=1 \mathrm{~nm}$ around the apex (small features) to $\Delta_{\mathrm{r}}=\Delta_{\mathrm{z}}=15 \mathrm{~nm}$ elsewhere. One note here that the transition between the two areas must be meshed gradually (over 400 spatial nodes) in order to cancel parasitical numerical reflections. On the other hand, the dispersion of gold is described by a Drude-Lorentz model [43] that was especially adapted and implemented into the BOR-FDTD algorithm in addition to the perfectly matched layers technique for the absorbing boundary conditions.

The tip apex has a semispherical shape of radius $R$. A radially polarized Bessel beam is supposed to illuminate the end part of the metallic thin tip at normal incidence (see Fig. 1a). It consists on a temporally Gaussian pulse centered on $\lambda=580 \mathrm{~nm}$ and having enough small duration to cover all the spectral range from 400 to $2,000 \mathrm{~nm}$. Its spatial shape is also Gaussian 
Fig. 1 Theoretical model of the structured tip in (a) and the proposed experimental setup to obtain the used peculiar illumination in (b)
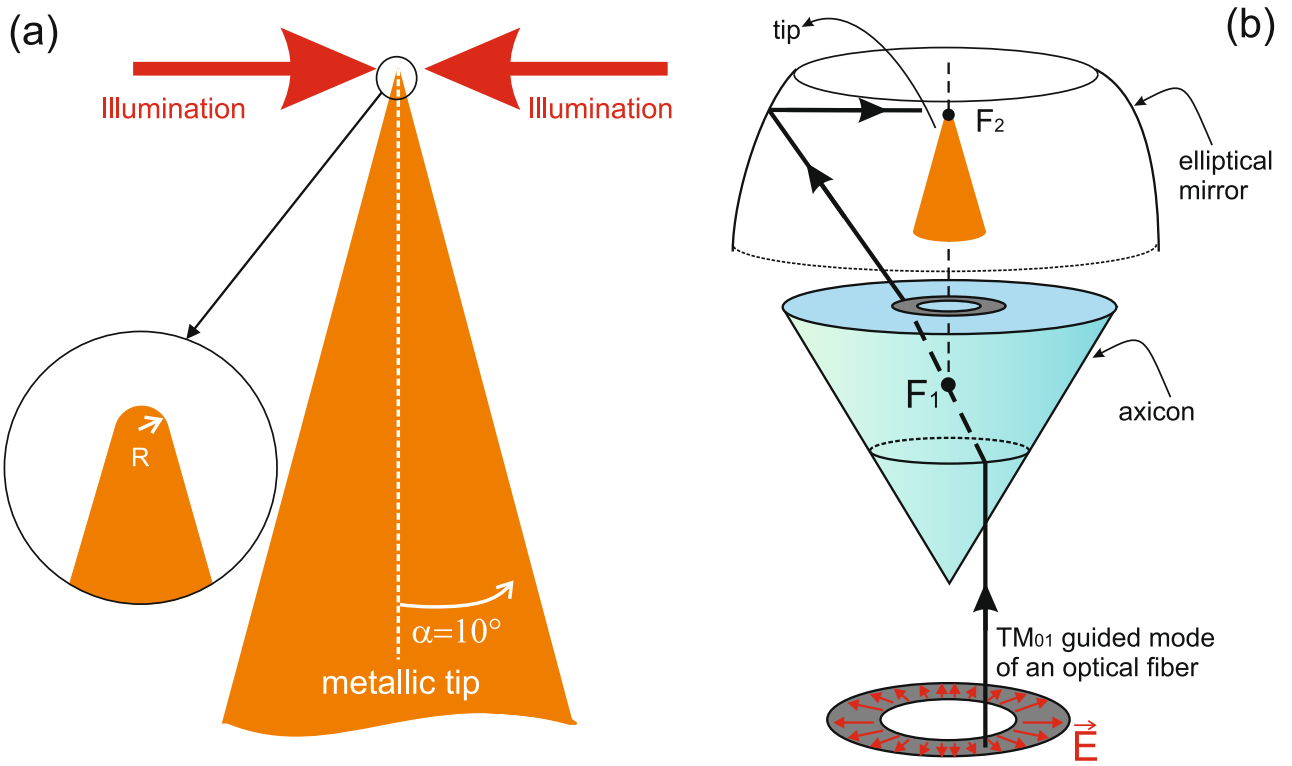

with $10 \mu \mathrm{m}$ beam-waist centered on the tip apex. This kind of beams can directly be generated with a classical optical fiber [44]. In order to be more realistic, we present in Fig. 1b a possible way to generate such an illumination: the $\mathrm{TM}_{01}$-guided mode of an optical fiber is sent through an axicon. By means of an elliptical mirror, the exit light is directed and focused on the tip. At this stage, one can think that this theoretical scheme is very complex and needs a very high accuracy for the alignment. However, a quite similar scheme was already experimentally built and used as demonstrated in [45] (see Figure 6c of that paper).

The confinement $\chi$ is defined as the electric field amplitude measured at a fixed point in front of the tip (here at $5 \mathrm{~nm}$ ) divided by the same quantity calculated without the tip. Figure 2 presents the obtained spectra
Fig. 2 Spectral responses of the enhancement factor $\chi$ for different tip radii (varying from 5 to $50 \mathrm{~nm}$ by step of $5 \mathrm{~nm}$ ) and for different metals: perfect conductor in (a), gold in (b), and silver in (c). d Three responses at a fixed radius value $(R=5 \mathrm{~nm})$ in addition to the one obtained for a tip made in glass. In all cases, the cone angle of the tip is set to $\alpha=10^{\circ}$ and the illumination is centered on the tip apex
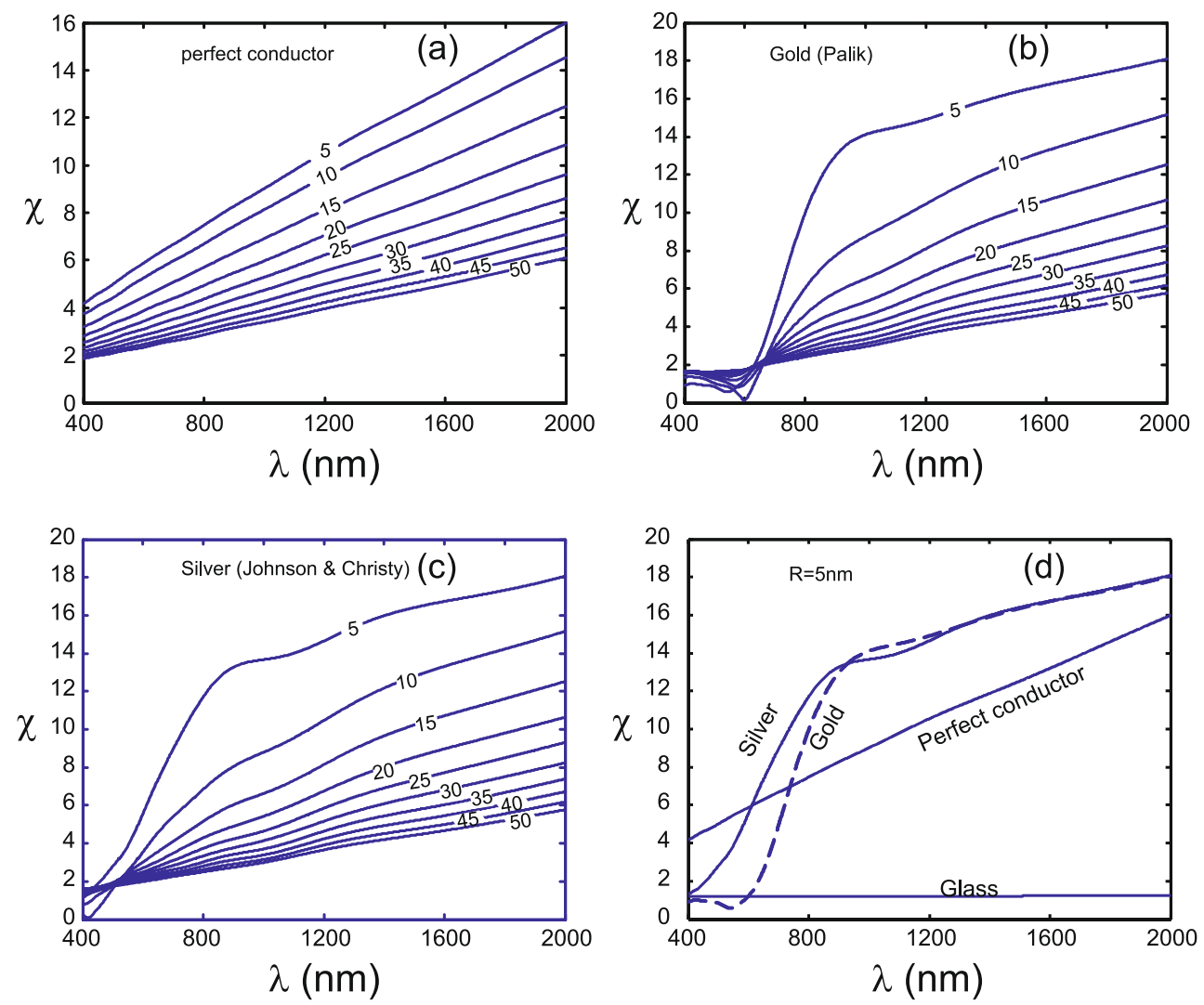
when the radius of the apex grows from $R=5 \mathrm{~nm}$ to $R=50 \mathrm{~nm}$ for tips made in perfect conductor (a), in gold (b), and in silver (c). For all cases, the evolution of the spectral responses is qualitatively similar except the fact that it almost presents a linear behavior in the case of perfect conductor. The case of silver seems to be more adapted to the visible region because it allows large value of $\chi$ for small tip radius.

It is clear form Fig. 2 that the antenna effect exists in the two cases of perfect or real metal. In the case of a perfect conductor, the confinement grows almost linearly with the wavelength and it increases when the apex radius decreases. For gold, the antenna effect is also obtained but only for wavelengths larger than $600 \mathrm{~nm}$. As for perfect conductor, the field enhancement has the same behavior with the apex radius except the presence of small oscillations appearing for large values of the wavelength.

The results of Fig. 2 are in good agreement with the electrostatic approach made in [38]. In fact, the key parameter is the ratio $R / \lambda$ that has to be very small in order to fulfill the electrostatic approach. For the visible range and in order to get valuable confinement coefficients (large than 10), the radius has to be smaller than $10 \mathrm{~nm}$ (see Fig. 2d). Consequently, we have fixed the tip radius to $5 \mathrm{~nm}$.

Note here that the results of Fig. 2 are obtained with a spatially finite illumination and by considering a very large tip length. So, these calculations are rather original because they are based on a modeling which almost respects faithfully the experimental parameters. This study of the antenna effect can be completed by determining the influence of, the cone angle, the spatial extension of the illumination and/or the metal nature in order to maximize the enhancement factor.

\section{Study of the structured tip}

In order to generate the radially polarized surface plasmon-guided mode, we proposed to corrugate the metallic tip at $10 \mu \mathrm{m}$ far from its end. In fact, and in addition to what is proposed in [26], our idea consists not only on the excitation of the SP mode but also on the use of such a guided mode to induce the antenna effect without the direct illumination of the tip apex. So, the incident beam directly illuminates corrugations (simple groove or conical grating) that are introduced on the walls of the metallic tip. The role of these features is to generate, by light diffraction, surface-guided modes on the conical face of the tip. As described in [24], a real metallic cylinder (with losses) has a radially polarized guided mode without cutoff. This means that when generated, this mode can, propagate along the tip walls whatever is the value of the radius, reach the apex and induce the antenna effect because the associated electric field presents a nonzero axial component.

In order to confirm these assumptions, we consider a conical metallic tip with an apex radius of $R=5 \mathrm{~nm}$ and a half-cone angle of $\alpha=10^{\circ}$. The corrugations consist, first, of a simple annular groove of 150-nm width and 200-nm depth located at $L_{1}=27.5 \mu \mathrm{m}$ from the apex (see Fig. 3a). The width of the groove is chosen to be smaller than $\lambda_{\min } / 2\left(\lambda_{\min }=400 \mathrm{~nm}\right.$ being
Fig. 3 Theoretical model of the structured tip with a groove (a) or with a finite grating (b) (a)

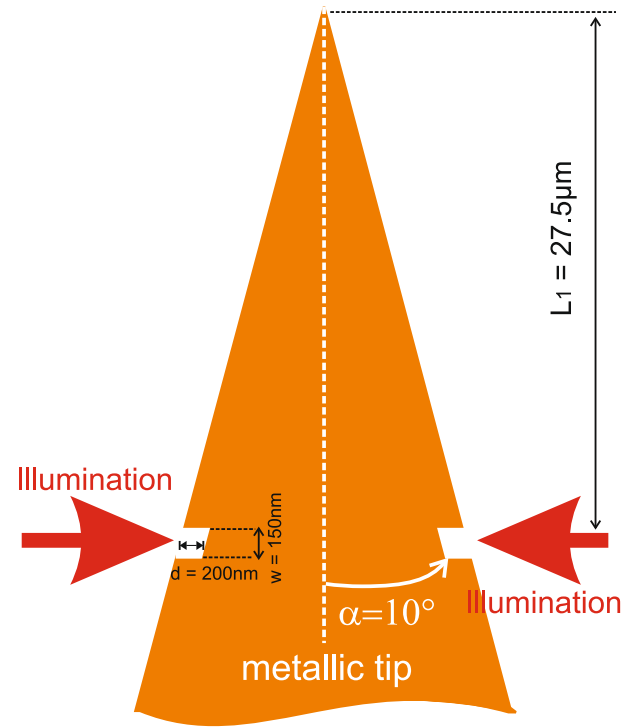

(b)

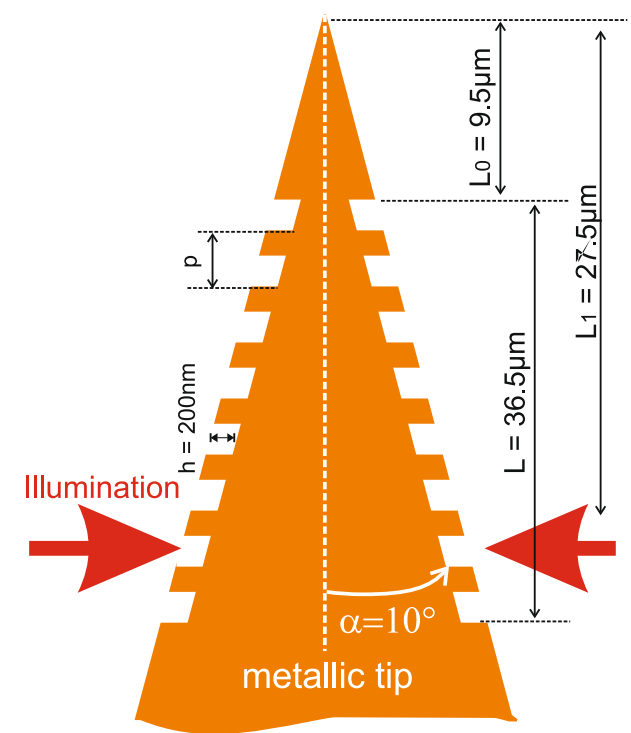


the smaller value of the wavelength for the considered spectral domain, i.e., [400 nm; 2,000 nm]) in order to get evanescent diffracted waves that can excite the SPP mode. The incident beam is centered at the groove and the detector is placed in front of the tip at $5 \mathrm{~nm}$ from the apex. In order to quantify the light enhancement at the apex we calculate the parameter $\tau$ which is the ratio of the electric field amplitude recorded by the detector by the same quantity measured at the center of the incident field (the Bessel Beam center that is at $L_{1}$ from the apex).

Calculations are performed for gold and silver metals. For tip made in gold, two Drude-Lorentz models were used to simulate the dispersion of this metal according to the experimental data of [46, 47]. The calculated spectral responses are presented in Fig. 4. It is clearly shown that, the enhancement depends on the antenna effect obtained with the same metal. In fact, for gold metal, efficient enhancements are only obtained for wavelengths larger than $600 \mathrm{~nm}$ due to the antenna effect that is prohibited below this value as shown in Fig. 2.

A flat response is obtained over $\lambda \in[1,000$; $1,500] \mathrm{nm}$ for gold and $\lambda \in[500 ; 1,500] \mathrm{nm}$ for silver. This broadband results from the compensation of two opposite phenomena: the antenna effect and the diffraction efficiency. The first one increases almost linearly while the second decreases exponentially when the wavelength increases. Beyond this plateau, the diffraction dominates and the amplitude falls down. Besides these two parameters, the decay length of the SPP intervenes on the enhancement factor because it depends on the wavelength value through the dispersion properties of the metal.

Figure 4 clearly shows that, with the considered geometry, it is only possible to get valuable enhancement in the visible range with the silver tip. For applications in nanooptics, the geometry must ideally enable the plateau obtained in Fig. 4, but over the visible region. This could be possible by modifying the metal nature and/or the probe cone angle.

For some other applications (SERS, Raman, or SHG), it is useful if the tip exhibits a spectral selectivity instead of a flat spectral response. This selectivity can be obtained by the use of a grating that enables the generation of the SPP around a desired wavelength value. So, we consider a finite grating of grooves (depth is fixed to $h=200 \mathrm{~nm}$ ) with a spatial extension limited to $L_{0}=9.5 \mu \mathrm{m}$ from the tip apex (cf. Fig. 3a). The total spatial length of the grating is $L=36.5 \mu \mathrm{m}$. As in the case of the single groove, the incident beam imping the grating at normal incidence and it is located at $27.5 \mu \mathrm{m}$ from the tip apex. Because of the axial symmetry, the interaction of light with the grating leads to diff racted orders with the same cylindrical symmetry. The propagation direction of each one is quantified through the usual grating formula:

$k_{/ /}^{\mathrm{d}}-k_{/ /}^{\mathrm{i}}=\frac{2 m \pi}{p_{/ /}}$

where $m$ is an integer, $k_{/ /}^{\mathrm{i}}$ and $k_{/ /}^{\mathrm{d}}$ are tangential (along the tip wall) components of the incident and diffracted wavevectors and $p_{/ /}$is the grating period along the tangential direction $\left(p_{/ /}=p / \cos \alpha\right)$.
Fig. 4 Enhancement factor of the electric field amplitude in the case of an annular groove of 150-nm width and 200-nm depth

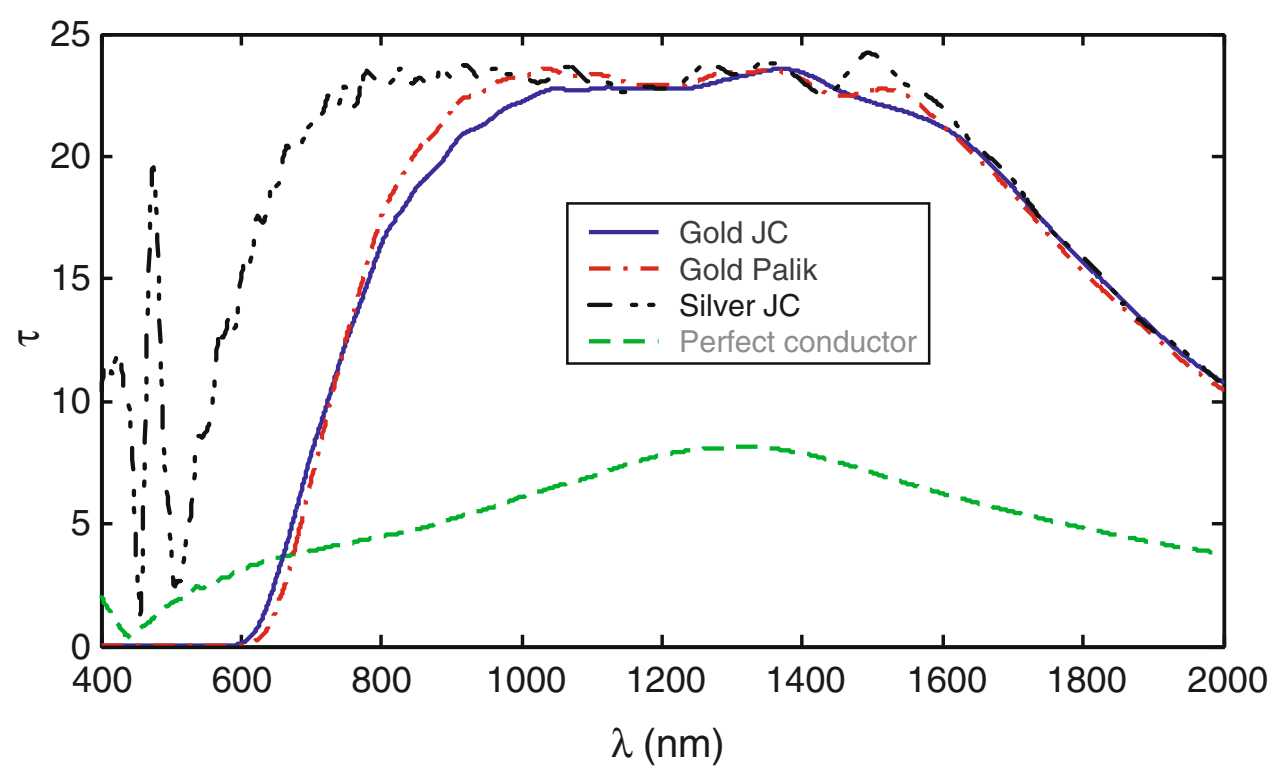


The SPP excitation occurs for diffracted wavevector $k_{/ /}^{\mathrm{d}}$ that matches the one of the surface plasmon mode $\left(k^{\mathrm{SPP}}\right)$. This latter is given by:

$k^{\mathrm{SSP}}=\frac{2 \pi}{\lambda} \sqrt{\frac{\varepsilon_{\mathrm{m}}(\lambda)}{\varepsilon_{\mathrm{m}}(\lambda)+1}}$

in the case of a tip surrounded by vacuum. This last equation corresponds to an SPP that is excited on a flat metallic interface not on a conical one. It still is valid because of the perfect cylindrical symmetry of both the object (tip with corrugations) and the illumination (the radially polarized beam).

According to these two equations (Eqs. 1 and 2), it is clear that the metal permittivity plays a key role in the determination of the SPP resonance. For a perfect conductor, where the permittivity tends to infinity, the SPP excitation occurs for $k_{/ /}^{\mathrm{d}}=\frac{\omega}{c}$ which corresponds to the Rayleigh anomaly. In the visible range, the metal permittivity becomes finite with very big real part comparing to its imaginary part. The SPP tangential wave-vector component, that is given by Eq. 2, becomes larger than $\frac{\omega}{c}$. So, as it is well-known, it consists on an evanescent wave.

By injecting Eq. 2 into Eq. 1, one can write:

$\lambda_{\mathrm{SPP}}=\frac{p}{m \cos \alpha}\left[\sqrt{\frac{\varepsilon_{\mathrm{m}}(\lambda)}{\varepsilon_{\mathrm{m}}(\lambda)+1}}-\sin \alpha\right]$

$\lambda_{\mathrm{SPP}}$ is here the wavelength for which the SPP is excited though diffraction by the grating and $\varepsilon_{\mathrm{m}}$ is the dielectric permittivity of the metal. By using the Drude-Lorentz model given in [43], we can calculate $\lambda_{\text {SPP }}$ for different period values (see Table 1 ).

We performed FDTD simulations with different period values and we calculated the enhancement factor $\chi$ in front of a gold tip. The obtained normalized spectra (the normalization is done as in Fig. 4) are presented in Fig. 5. They are recorded at $5 \mathrm{~nm}$ in front of a gold tip. Except the period value that is varied from 770 to $1,130 \mathrm{~nm}$, the geometrical parameters of the tips are fixed as indicated in Fig. 3. One can see that the spectra become thinner then those of Fig. 4 and the position of their maxima greatly depends on the period value. Consequently, the grating is playing its basic and fundamental role of a spectral filter based on the generation of the guided SPP mode of the tip.

The electromagnetic field that is confined in front of the tip apex (given by the $\chi$ factor value) is then great enough to induce nonlinear effects $[48,49]$ and can be used as a very focused and localized source in optical characterization of nanophotonic devices and to generate multiphoton electron emission [50].

Let us notice here that theoretical values of $\lambda_{\text {SPP }}$ given in Table 1 (second line) do not correspond exactly to the maxima of the spectra of Fig. 5 given in the third line of the same table. This discrepancy is probably due to the influence of the spectral response of the antenna effect (see Fig. 2) and could induce a small blue shift on the global spectral response of the tip only for small values of the period $(p<1,000 \mathrm{~nm})$. In addition and because of the spatial discretization used in the FDTD algorithm, the period value is defined with an uncertainty equal to the half of the spatial step value used to describe the grating (here $15 / 2=7.5 \mathrm{~nm}$ ). Consequently, the spectra are somewhat widened and the peak positions are slightly modified. In order to get more precise results, it is necessary to decrease the spatial mesh step and, accordingly, to make an arduous calculations that cannot be supported by our own computers.

Let us mention here that the spectral responses plotted in Figure 3 of [26] are in good agreement with spectra of Fig. 4. The intense local light spot, obtained in that study, is not only due to the excitation of the SPP-guided mode but also thanks to the antenna effect.

To end this prospective study, we present in Fig. 6 the light distributions for two different values of the wavelength corresponding to a gold tip with (at $\lambda=$ $756 \mathrm{~nm}$ ) and without (for $\lambda=1,400 \mathrm{~nm}$ ) SPP excitation. The period is set to $p=900 \mathrm{~nm}$ and the apex radius is $R=5 \mathrm{~nm}$. In (a) and (c), the SPP is excited at $\lambda=765 \mathrm{~nm}$ leading to a large enhancement factor while this last almost disappears at $\lambda=1,400 \mathrm{~nm}$ in (b) and (d). Because of the large spatial confinement of the electric field in (a) and (c), the fifth root of the square

Table 1 Theoretical values of the wavelengths that correspond to the excitation of the SPP with respect to the corrugation period value calculated from Eq. 3

\begin{tabular}{|c|c|c|c|c|c|c|c|c|c|c|c|c|c|}
\hline$p(n m)$ & 770 & 800 & 830 & 860 & 890 & 920 & 950 & 980 & 1,010 & 1,040 & 1,070 & 1,100 & 1,130 \\
\hline$\lambda_{\mathrm{SPP}}(\mathrm{nm})$ & 674.2 & 697.16 & 720.5 & 744 & 767.8 & 791.75 & 815.85 & 840.1 & 864.4 & 888.8 & 913.3 & 927.84 & 962.44 \\
\hline$\lambda_{\mathrm{SPP}}^{\mathrm{FDTD}}(\mathrm{nm})$ & 668 & 694 & 718 & 741 & 764 & 788 & 808 & 834 & 862 & 879 & 915 & 936 & 961 \\
\hline
\end{tabular}

The third line gives the position of the maxima of the spectra presented in Fig. 5 and calculated by FDTD 
Fig. 5 Calculated

enhancement factor spectra for the 13 values of the grating period of Table 1 .

The considered tip is made in gold and its geometry is completely defined in Fig. $3 b$ with an apex radius $R=5 \mathrm{~nm}$

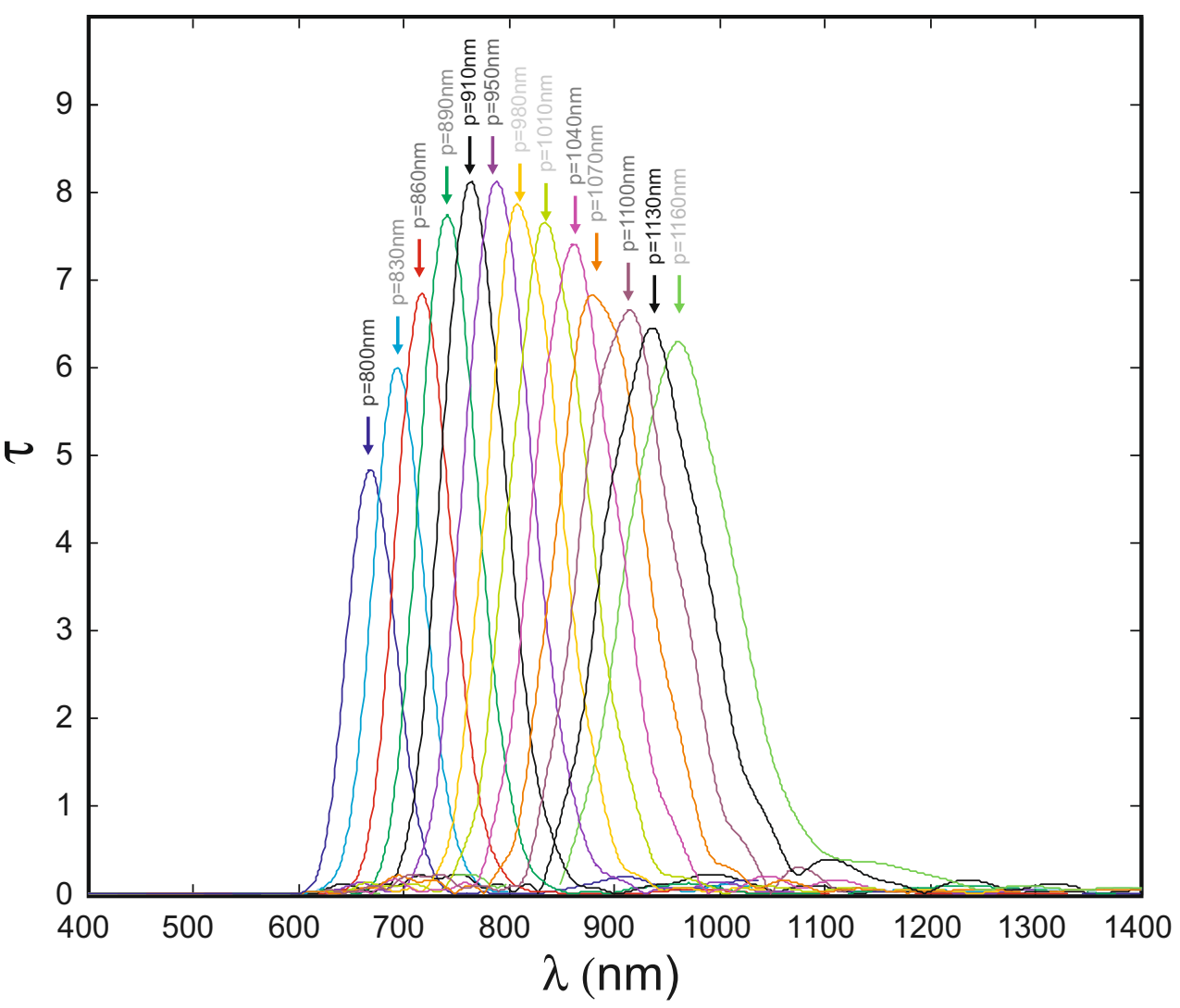

modulus of the electric field is presented instead of the electric intensity. In spite of that, the incident beam cannot be clearly seen in Fig. 6a because of the very big light enhancement at the apex. However, interferences between incident and reflected beams are clearly visible in Fig. 6b.
Fig. 6 Light intensity distributions in color level (fifth root of the square modulus of the electric field). a, b For the whole calculation domain, c, $\mathbf{d}$ are zoom-in made around the tip apex on (a) and (b), respectively. The SPP is excited in (a) and (c) at $\lambda=756 \mathrm{~nm}$ while it disappears in (b) and (d) at $\lambda=1,400 \mathrm{~nm}$. The grating period is set to $p=900 \mathrm{~nm}$

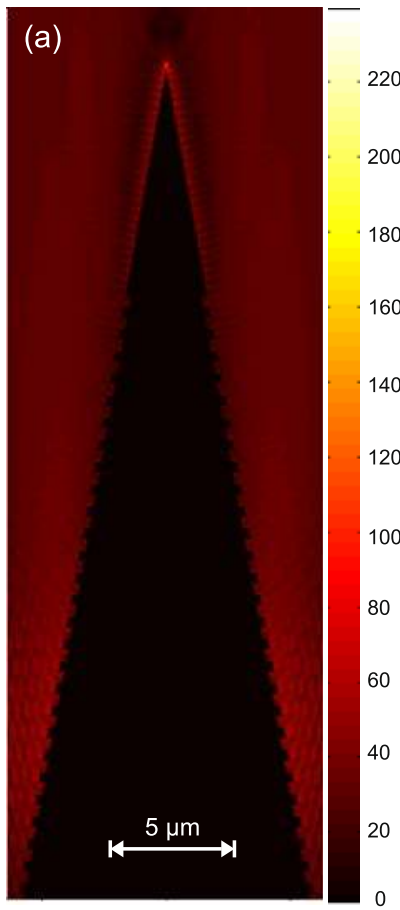

(c)

220

60

20

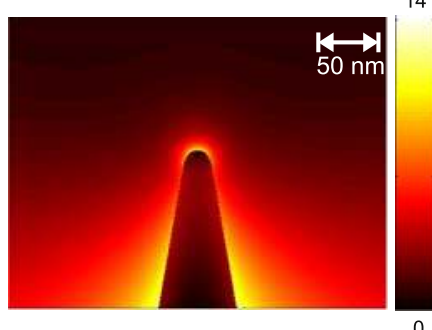




\section{Conclusion}

In summary, we have proposed and theoretically demonstrated a new configuration based on a conical metallic tip exhibiting a very high confinement of light. A super-focusing effect is obtained at the tip end even if this last one is not enlightened. This was possible thanks to the combination of two phenomena: the excitation of a guided SPP mode at a rather big distance from the tip apex with the antenna effect. The major and important point to be noted is that this SPP mode can reach the apex because it has no cutoff. Thus, if other modes are generated (due to misalignment in the experience), they cannot affect the tip end and will give rise to radiative waves. The next step of this work consists on the experimental fabrication and characterization of such a probe. Works are in progress to fabricate such tips in gold or silver through the focused ion beam etching technique.

Acknowledgements This work has been partially supported by C'Nano GE, the Pôle de Compétences en Nanosciences Grand Est.

\section{References}

1. Hecht B, Bielefeldt H, Inouye Y, Pohl DW, Novotny L (1997) Facts and artifacts in near-field optical microscopy. J Appl Phys 81(6):2492-2498, March

2. Hecht B, Bielefeldt H, Pohl DW, Novotny L, Heinzelmann H (1998) Influence of detection conditions on near-field optical imaging. J Appl Phys 84(11), 5873-5882, December

3. Chavez-Pirson A, Temmyo J, Kamada H, Gotoh H, Ando H (1998) Near-field optical spectroscopy and imaging of single ingaas/algaas quantum dots. Appl Phys Lett 72:3494

4. Ono M, Matsuda K, Saiki T, Nishi K, Mukaiyama T, Kuwata-Gonokami M (1999) Time-resolved emission from self-assembled single quantum dots using scanning near-field optical microscope. Jpn J Appl Phys 38:L1460

5. Vohnsen B, Bozhevolnyia SI, Pedersena K, Erland J, Jensenb JR, Hvam JM (2001) Second-harmonic scanning optical microscopy of semiconductor quantum dots. Opt Commun 189:305

6. Kawazoe T, Kobayashi K, Akahane K, Naruse M, Yamamoto N, Ohtsu M (2006) Demonstration of nanophotonic not gate using near-field optically coupled quantum dots. Appl Phys B 84:243

7. Phillips PL, Knight JC, Mangan BJ, Russell PStJ, Charlton MDB, Parker GJ (2006) Near-field optical microscopy of thin photonic crystal films. Appl Phys Lett 85:6337 (1999)

8. Koenderink AF, West R, Buchler BC, Richter S, Strasser P, Kafesaki M, Rogach A, Wehrspohn RB, Soukoulis CM, Erni D, Robin F, Jäckel H, Sandoghdar V (2005) Near-field optics and control of photonic crystals. Photonics and Nanostructures: Fundamentals and Applications 3:63

9. Bernal M-P, Courjal N, Amet J, Roussey M, Hou CH (2006) Lithium niobate photonic crystal waveguides, far field and near field characterisation. Opt Commun 265:180
10. Martin MJ, Benyattou T, Orobtchouk R, Talneau A, Berrier A, Mulot M, Anand S (2006) Evidence of bloch wave propagation within photonic crystal waveguides. Appl Phys B 82:9-12

11. Bozhevolnyi SI, Kuipers L (2006) Near-field characterization of photonic crystal waveguides. Semicond Sci Technol 21:R1-R16

12. Lalouat L, Cluzel B, Velha P, Picard E, Peyrade D, Hugonin JP, Lalanne P, Hadji E, de Fornel F (2007) Near-field interactions between a subwavelength tip and a small-volume photonic-crystal nanocavity. Phys Rev B 76:041102

13. Sandtke M, Engelen RJP, Schoenmaker H, Attema I, Dekker H, Cerjak I, Korterik JP, Segerink FB, Kuipers L (2008) Novel instrument for surface plasmon polariton tracking in space and time. Rev Sci Instrum 79:013704

14. Sandoghdar V, Mlynek J (1999) Prospects of apertureless snom with active probes. J Opt A, Pure Appl Opt 1:523-530

15. Hayazawa N, Inouye Y, Sekkat Z, Kawata S (2000) Metallized tip amplification of near-field raman scattering. Opt Commun 183:333-336

16. Kramer A, Trabesinger W, Hecht B, Wild UP (2002) Optical near-field enhancement at a metal tip probed by a single fluorophore. Appl Phys Lett 80(9):1652

17. Laurent G, Felidj N, Grand J, Aubard J, Levi G, Hohenau A, Aussenegg FR, Krenn JR (2006) Raman scattering images and spectra on gold ring arrays. Phys Rev B 73:245417

18. Kawata S, Shalaev VM (2006) Advances in nano-optics and nano-photonics. Elsevier, Amsterdam

19. Kramer A, Keilmann F, Knoll B, Guckenberger R (1996) The coaxial tip as a nano-antenna for scannng near-field microwave transmission microscopy. Micron 27:413-417

20. Leinhos T, Rudow O, Stopka M, Vollkopf A, Oesterschulze E (1999) Coaxial probes for scanning near-field microscopy. J Microsc 194:349-352

21. Sqalli O, Bernal M-P, Hoffmann P, Marquis-Weible F (2000) Improved tip performance for scanning near-field optical microscopy by the attachment of a single gold nanoparticle. Appl Phys Lett 76(15):2134-2136

22. Keilmann F (2002) Vibrational-infrared near-field microscopy. Vibr Spectrosc 29:109-114

23. Antosiewicz TJ, Szoplik T (2007) Corrugated metal coated tapered tip for scanning near field optical microscope. Opt Express 15:10920-10928

24. Ding W, Andrews SR, Maier SA (2007) Internal excitation and superfocusing of surface plasmon polaritons on a silvercoated optical fiber tip. Phys Rev A 75:063822

25. Chen W, Zhan Q (2007) Numerical study of an apertureless near field scanning optical microscope probe under radial polarization illumination. Opt Express 17(7):4106

26. Ropers C, Neacsu CC, Elsaesser T, Albrecht M, Raschke MB, Lienau C (2007) Grating-coupling of surface plasmons onto metallic tips: a nanoconfined light source. Nano Lett 7(9):2784-2788

27. Issa NA, Guckenberger R (2007) Optical nanofocusing on tapered metallic waveguides. Plasmonics 2:31-37

28. H'Dhili F, Bachelot R, Lerondel G, Barchiesi D, Royer P (2001) Near-field optics: direct observation of the field enhancement below an apertureless probe using a photosensitive polymer. Appl Phys Lett 79:4019-4021

29. Fikri R, Barchiesi D, H'Dhili F, Bachelot R, Vial A, Royer $\mathrm{P}$ (2003) Modeling recent experiments of apertureless nearfield optical microscopy using $2 \mathrm{~d}$ finite element method. Opt Commun 221:13-22

30. Bachelot R, H'Dhili F, Barchiesi D, Lerondel G, Fikri R, Royer P, Landraud N, Peretti J, Chaput F, Larnpel G, Boilot JP, Lahlil K (2003) Apertureless near-field optical microscopy: a study of the local tip field enhancement using 
photosensitive azobenzene-containing films. J Appl Phys 94:2060-2072

31. Bohn JL, Nesbitt DJ, Gallagher A (2001) Field enhancement in apertureless near-field scanning optical microscopy. J Opt Soc Am A 18(12):2998

32. Calander N, Willander M (2002) Theory of surface-plasmon resonance optical-field enhancement at prolate spheroids. J Appl Phys 92:4878

33. Krug JT II, Sanchez EJ, Sunney Xiea X (2002) Design of near-field optical probes with optimal field enhancement by finite difference time domain electromagnetic simulation. J Chem Phys 116:10895-10901

34. Renger J, Grafström S, Eng LM, Deckert V (2004) Evanescent wave scattering and local electric field enhancement at ellipsoidal silver particles in the vicinity of a glass surface. J Opt Soc Am A 21:1362

35. Roth RM, Panoiu NC, Adams MM, Osgood RM, Neacsu CC, Raschke MB (2006) Resonant-plasmon field enhancement from asymmetrically illuminated conical metallic-probe tips. Opt Express 14(7):2921

36. Cui X, Zhang W, Yeo B-S, Zenobi R, Hafner C, Erni D (2007) Tuning the resonance frequency of ag-coated dielectric tips. Opt Express 15:8309

37. Denk W, Pohl DW (1991) Near-field optics: microscopy with nanometer-size fields. J Vac Sci Technol, B 9:510

38. Demming F, Jersch J, Dickmann K, Geshev PI, Muenster FH (1998) Calculation of the field enhancement on laserilluminated scanning probe tips by the boundary element method. Appl Phys B 66:593

39. Grosjean T, Baida F, Courjon D (2007) Conical optics: the solution to confine light. Appl Opt 46:1994-2000

40. Davidson DB, Ziolkowski RW (1994) Body-of-revolution finite-difference time-domain modeling of space-time focusing by a three-dimensional lens. J Opt Soc Am A 11(4):14711490, April
41. Parent G, Van Labeke D, Baida FI (2001) Theoretical study of transient phenomena in near-field optics. J Microsc 202(2):296-306, May

42. Baida FI, Van Labeke D, Pagani Y (2003) Body-ofrevolution FDTD simulations of improved tip performance for scanning near-field optical microscopes. Opt Commun 255:241-252

43. Vial A, Grimault A-S, Macas D, Barchiesi D, de la Chapelle ML (2005) Improved analytical fit of gold dispersion: application to the modeling of extinction spectra with a finitedifference time-domain method. Phys Rev B 71:085416

44. Grosjean T, Courjon D, Spajer M (2002) An all-fiber device for generating radially and other polarized light beams. Opt Commun 203(1-2):1-5, March

45. Bainier C, Vannier C, Courjon D, Rivoal J-C, Ducourtieux S, De Wilde Y, Aigouy L, Formanek F, Belliard L, Siry P, Perrin B (2003) Comparison of test images obtained from various configurations of scanning near-field optical microscopes. Appl Opt 42(4), 691-700

46. Palik E (1985) Handbook of optical constants of solids. Academic, New York

47. Johnson PB, Christy RW (1972) Optical constants of the noble metals. Phys Rev B 6:4370-4379

48. Bouhelier A, Beversluis M, Hartschuh A, Novotny L (2003) Near-field second-harmonic generation induced by local field enhancement. Phys Rev Lett 90:013903

49. Laroche T, Baida FI, Van Labeke D (2005) Threedimensional finite-difference time-domain study of enhanced second-harmonic generation at the end of a apertureless scanning near-field optical microscope metal tip. J Opt Soc Am B 22:1045-1051

50. Ropers C, Solli DR, Schulz CP, Lienau C, Elsaesser $\mathrm{T}$ (2007) Localized multiphoton emission of femtosecond electron pulses from metal nanotips. Phys Rev Lett 98: 043907 